\title{
MicroRNA-210 promotes cancer angiogenesis by targeting fibroblast growth factor receptor-like 1 in hepatocellular carcinoma
}

\author{
YUN YANG $^{1 *}$, JIN ZHANG $^{1 *}$, TIAN XIA $^{1-3^{*}}$, GAIYUN LI $^{2 *}$, TAO TIAN $^{1}$, MENGCHAO WANG $^{1}$, \\ RUOYU WANG ${ }^{1}$, LINGHAO ZHAO ${ }^{1}$, YUAN YANG ${ }^{1}, \mathrm{KE} \mathrm{LAN}^{2}$ and WEIPING ZHOU ${ }^{1}$ \\ ${ }^{1}$ The Third Department of Hepatic Surgery, Eastern Hepatobiliary Surgery Hospital, Shanghai 200438; \\ ${ }^{2}$ The Key Laboratory of Molecular Virology and Immunology, Institute Pasteur of Shanghai, \\ Chinese Academy of Sciences, Shanghai 200031, P.R. China; \\ ${ }^{3}$ Department of Virology, Institute Pasteur, Paris 75015, France
}

Received December 14, 2015; Accepted January 21, 2016

DOI: 10.3892/or.2016.5129

\begin{abstract}
Hypoxia drives cancer to become more aggressive, particularly angiogenesis, and the corresponding mechanisms still need to be further investigated. In hepatocellular carcinoma (HCC), the master hypoxia-induced microRNA (miRNA) miR-210 is upregulated in HCC and participates in HCC progression, but its roles in hypoxia-induced HCC angiogenesis are still unknown. Moreover, the correlation between miR-210 expression and HCC clinical progression also needs elucidation. In the present study, we found that miR-210 expression was progressively increased from normal liver and adjacent non-tumor tissues, to incipient and advanced tumor tissues. In HCC patients, high miR-210 expression was significantly correlated with poor prognosis, both tumor-free survival and overall survival. Moreover, miR-210 expression in HCC was significantly positively correlated with microvascular density. Both in vitro and in vivo studies determined that miR-210 promoted HCC angiogenesis, and the corresponding mechanism was identified to be the direct targeting and inhibition of fibroblast growth factor receptor-like 1 (FGFRL1) expression. Thus, we suggest a new prognosis predictor for HCC patients, and determined the roles of hypoxic miR-210 in HCC angiogenesis.
\end{abstract}

Correspondence to: Professor Weiping Zhou, The Third Department of Hepatic Surgery, Eastern Hepatobiliary Surgery Hospital, 225 Changhai Road, Shanghai 200438, P.R. China E-mail: ehphwp@126.com

Professor Ke Lan, The Key Laboratory of Molecular Virology and Immunology, Institute Pasteur of Shanghai, Chinese Academy of Sciences, Shanghai 200031, P.R. China

E-mail: lanke@sibs.ac.cn

${ }^{*}$ Contributed equally

Key words: miR-210, prognosis, angiogenesis, fibroblast growth factor receptor-like 1, hepatocellular carcinoma

\section{Introduction}

More than 350 million people are infected with chronic hepatitis B virus (HBV) worldwide, and are at risk of developing liver diseases, such as chronic hepatitis, cirrhosis and hepatocellular carcinoma (HCC) (1). Due to widespread use of the HBV vaccine, the global pandemic of HBV has decreased in prevalence, but the absolute number of HBsAg-positive individuals is still increasing (2). In addition, there are huge populations with chronic HBV infection, particularly in East Asia, and HBV-related liver diseases, particularly HCC. Hence, HBV-related liver diseases will continue to be a public health burden for decades.

Due to the rapid growth of cancer cells and limited oxygen from the environment, hypoxia is a common feature in cancer (3). In solid tumors such as HCC, the cancer cells encounter low oxygen pressure stress, particularly those cancer cells encompassed in tumor tissues (4). In order to adapt to hypoxic stress, cancer cells induce neovasculature, breaking the natural barriers to tumor angiogenesis (3), thus pushing cancer to be more aggressive. Hypoxic stress induces the expression of hypoxia-induced factor $1 \alpha$ (HIF-1 $\alpha)$, which translocates into the nucleus and acts as a transcription factor to initiate the expression of downstream genes and one master hypoxic microRNA (miRNA), miR-210 (5). miR-210 regulates hypoxia-induced intracellular pathways, including cell cycle progression (6-9), cell survival $(10,11)$, genome stabilization (12), cell differentiation $(13,14)$, angiogenesis $(15-24)$ and cell metabolism (25-27). The hypothesis that upregulated miR-210 may help cancer cells adapt to neoplastic stress has been partly proved by the increased potential of metastasis in HCC cells (28), and the increased cell growth, survival, genome destabilization and angiogenesis in other human tumor models (29). Moreover, miR-210 is a regulator of multiple function in terms of carcinogenesis and progression, and it can act as both oncogene and tumor suppressor under different conditions or in different types of cancer (30).

miRNAs are special non-coding RNAs, which are involved in a wide array of cellular processes, including 
differentiation, proliferation, apoptosis, stress, carcinogenesis and progression $(31,32)$. In recent years, miRNAs have been identified as important regulators in liver diseases, including HCC (33). Among these miRNAs, upregulation of miR-210 in HCC tumor tissues was first reported in genomic screening studies (34-36), and its upregulation in the serum of HCC patients was also reported (37). Functional studies showed that the upregulation of hypoxic miR-210 in HCC induced cell metastasis and radiotherapy resistance, although its role in cell proliferation was not consistent possibly due to different experimental approaches $(28,38,39)$. However, it remains unclear whether miR-210 contributes to HCC angiogenesis, and whether deregulation of miR-210 can be a new prognosis predictor for HCC patients.

In the present study, we examined the roles of miR-210 in the prognosis and angiogenesis of HBV-related HCC, using both clinical samples and cell models. We found that miR-210 expression is upregulated in HCC tumor tissues, and high expression of miR-210 in HCC tissues is an independent risk factor for both tumor-free and overall survival of HCC patients. In addition, the relative miR-210 expression in tumor/non-tumor of HCC patients may serve as a prognostic factor. Moreover, the present study provides evidence to show that miR-210 promoted HCC angiogenesis, and the corresponding mechanism was identified to be the direct targeting and inhibition of fibroblast growth factor receptor-like 1 (FGFRL1) expression. Thus, we suggested a new prognosis predictor for HCC patients, and determined the roles of hypoxic miR-210 in HCC angiogenesis.

\section{Materials and methods}

Patients. All clinical samples were obtained with informed consent from the HCC surgery undertaken in Eastern Hepatobiliary Surgery Hospital, Shanghai, China. From 2007 to 2010, 212 paired HCC and non-tumor samples from HBV-related HCC patients were examined, who were HBV-positive (HBsAg, HBV DNA or HBeAg serum-positive) and HCV-negative (anti-HCV serum-negative) (Table I). Thirty-one non-tumor tissues served as healthy liver controls, and were obtained from hepatic hemangioma patients, without HBV infection (serum HBsAg and anti-HBc-negative). The present study was approved by the Ethics Committee of Eastern Hepatobiliary Surgery Hospital.

Cells, plasmids, reagents and antibodies. Cells: HL-7702 and SMMC-7721 cells were maintained in RPMI-1640 medium (HyClone, Logan, UT, USA) supplemented with $10 \%$ fetal bovine serum (FBS; Gibco, Carlsbad, CA, USA). Huh7 and 293 T cells were maintained in Dulbecco's modified Eagle's medium (DMEM) (HyClone) supplemented with 10\% FBS. The cells were purchased from the Cell Bank of Institutes for Biological Sciences, Chinese Academy of Sciences, Shanghai. Primary human umbilical vein endothelial cells (HUVECs) were purchased from Lonza and were maintained in EGM2 medium (Lonza, Walkersville, MD, USA). Plasmids: the pre-miR-210 fragment was cloned from human hepatocyte genome into the pCDH-CMV-MCS-EF1-puro vector (SBI), for miR-210 lentivirus production and infection. Lentivirus package plasmids, CMV-VSVG and $\delta-8.9$, were purchased from SBI. Lentivirus production, infection and establishment of stable transduced cell lines were performed according to the manufacturer's protocol. Reagents: TRIzol agent (Invitrogen, Carlsbad, CA, USA), puromycin, deferoxamine (DFX) (both from Sigma, St. Louis, MO, USA), Matrigel ${ }^{\mathrm{TM}}$ Matrix (BD Biosciences, Bedford, MA, USA), recombinant mouse FGF R5/FGFRL1 Fc Chimera (R\&D Systems, Minneapolis, MN, USA). miR-210 mimic, mimic control, miR-210 inhibitor and inhibitor control (RiboBio, Guangzhou, China). BCA protein assay kit (Beyotime, Jiangsu, China) and Complete Protease Inhibitor Cocktail Tablets (Roche). Antibodies: anti-HIF1 $\alpha$, anti-FGFRL1 and anti-GAPDH (Sigma), anti-human and mouse CD34 (Abcam, Cambridge, MA, USA).

RNA extraction and miRNA $q R T-P C R$. Total RNA was isolated from clinical samples or cultured cells by TRIzol according to the manufacturer's protocol. The quality of the RNA was assessed by NanoDrop 2000 (NanoDrop Technologies, Wilmington, DE, USA). miRNA qRT-PCR was performed using the TaqMan Human miRNA Assay kit (Applied Biosystems, Foster City, CA, USA) according to the manufacturer's instructions. The expression of miR-210 was normalized relative to the expression of small nuclear RNA U6, as an internal control. Data were analyzed using the comparative $\mathrm{Ct}$ method $\left(2^{-\Delta \Delta \mathrm{Ct}}\right)$.

Protein purification and western blot (WB) assay. Secreted FGFRL1 protein in the culture medium was purified by heparin-sepharose as previously described (40). Briefly, heparin-sepharose was washed with phosphate-buffered saline (PBS) twice, and then added to PBS 1:1 diluted culture medium, the culture medium was filtered using a $0.45 \mu \mathrm{m}$ filter. Heparin-sepharose and the medium mixture were rotated at $4^{\circ} \mathrm{C}$ for $4 \mathrm{~h}$. Heparin-sepharose was pelleted using a centrifuge and washed 5 times with PBS. Heparin binding proteins were eluted with Laemmli buffer at $95^{\circ} \mathrm{C}$, and then subjected to SDS-PAGE for WB assay. For WB assay, the cells were lysed in lysis buffer (50 mM Tris, $\mathrm{pH} 8.0,150 \mathrm{mM} \mathrm{NaCl}$, $1 \%$ Nonidet P-40 and complete protease inhibitor mixture), for $30 \mathrm{~min}$ at $4^{\circ} \mathrm{C}$. The cell lysates were centrifuged at $12,000 \mathrm{x} \mathrm{g}$ and $4^{\circ} \mathrm{C}$ for $10 \mathrm{~min}$, and the supernatant was used in the next step. The cell lysates were quantified using the BCA kit and adjusted to the same level in each experiment. The protein samples were then subjected to SDS-PAGE and transferred to nitrocellulose membranes for WB assay.

Tube formation assay. The protocol for endothelial cell tube formation assay was based on the procedure outlined by Corning (Corning, NY, USA). Briefly, for pre-treatment, HUVEC cells were serum-starved $12 \mathrm{~h}$ before the tube formation assay, and the Matrigel ${ }^{\mathrm{TM}}$ Matrix was thawed on ice overnight. Thawed Matrigel Matrix (50 $\mu \mathrm{l})$ was added to each well of a pre-chilled 96-well sterile plate. The plate was incubated for $30 \mathrm{~min}$ at $37^{\circ} \mathrm{C}$ to allow the Matrigel Matrix to form a gel. HUVEC cells were harvested and re-suspended in the indicated medium at $1.5 \times 10^{5}$ cells $/ \mathrm{ml}$. Cell suspension $(100 \mu \mathrm{l})$ $\left(1.5 \times 10^{4}\right.$ cells) was added to each well containing solidified Matrigel Matrix. The assay plate was incubated at $37^{\circ} \mathrm{C}$ for $4 \mathrm{~h}$. The endothelial tubes were pictured for calculation using the angiogenesis-analyzer tool plugin for ImageJ. 
A

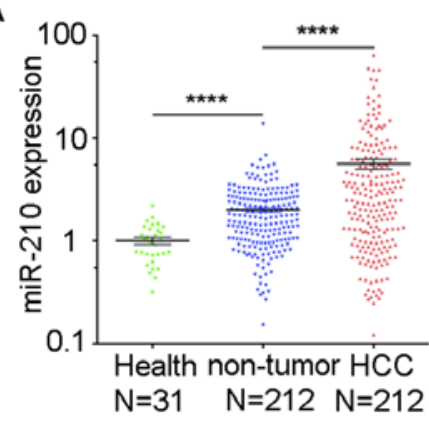

B

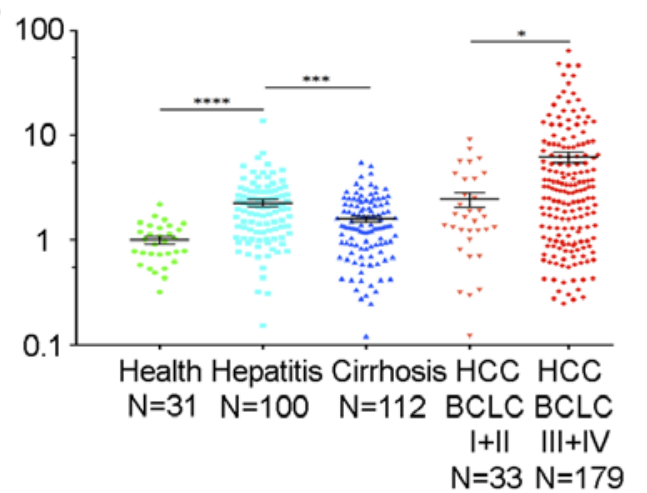

D

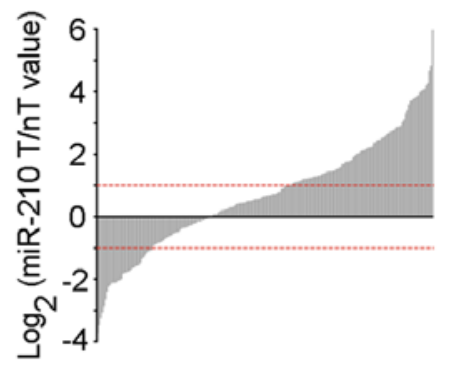

C

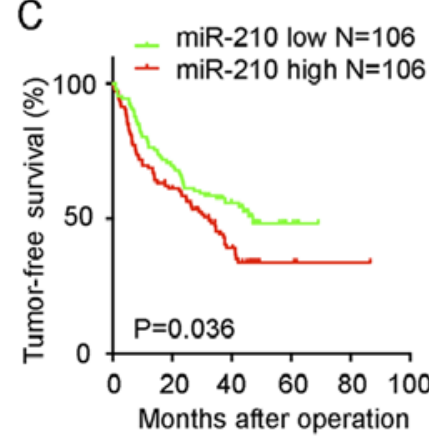

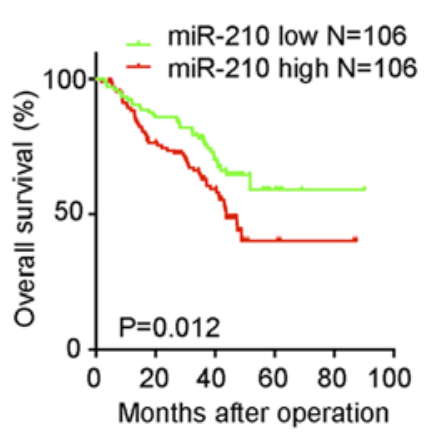

E

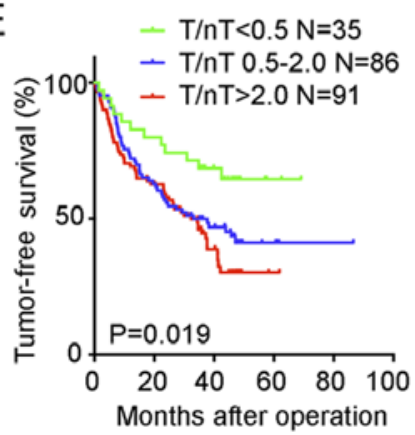

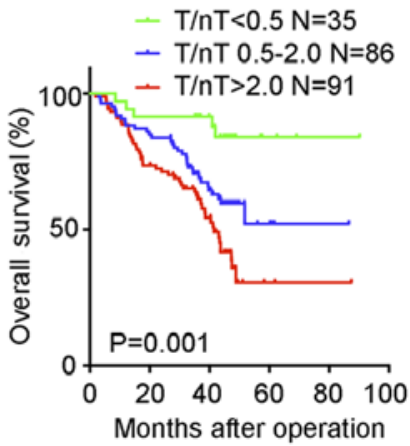

Figure 1. Increased miR-210 in HCC tissues is correlated with prognosis. (A) miR-210 expression levels in non-HBV healthy liver tissues (n=31) are significantly lower than those in HBV-related HCC non-tumor tissues $(\mathrm{n}=212 ; \mathrm{P}<0.001)$. miR-210 expression levels in HBV-related HCC tumor tissues are significantly higher than those in paired non-tumor controls $(n=212 ; \mathrm{P}<0.001)$. (B) miR-210 expression levels at different disease stages. miR-210 expression in hepatitis non-tumor tissue $(n=100)$ was significantly higher than that in healthy control liver $(n=31)$ and cirrhotic liver tissue $(n=112)(P<0.0001 ; P=0.0004$, respectively). In tumor tissue, miR-210 expression was significantly higher in late-stage tumors $(\mathrm{P}=0.0297)$. (C). Kaplan-Meier analysis of tumor-free survival and overall survival based on miR-210 levels in HCC tissues of 212 HBV-related HCC patients. Patients with low miR-210 levels in HCC tissues had a better clinical outcome than patients with high miR-210 levels $(\mathrm{P}=0.036 ; \mathrm{P}=0.012)$. (D) miR-210 expression T/nT value in HCC patients. (E) Kaplan-Meier analysis of tumor-free survival and overall survival based on miR-210 T/nT value in $212 \mathrm{HBV}$-related HCC patients. The patients with relatively low miR-210 T/nT value had a better clinical outcome than patients with high miR-210 T/nT value $(\mathrm{P}=0.019 ; \mathrm{P}=0.0001)$.

Microvesseldensity (MVD)CD34assay.Immunohistochemical staining for CD34 in both human and mouse liver sections was performed in the Immunohistochemistry Department of Eastern Hepatobiliary Surgery Hospital. The MVD CD34 value was assessed using the method developed by Weidner et al (41). Briefly, the CD34 immunohistochemically stained tissue sections were examined in the 200x field, 5 hotspots were pictured for MVD assessment. Any endothelial cell or endothelial-cell cluster stained brown which was clearly separate from adjacent microvessels, tumor cells and other connective tissue elements was considered a single countable microvessel. The average of the 5 hotspots in each sample was considered the MVD CD34 value.

Xenograft growth. miR-210 stably transduced SMMC-7721 and control cells were trypsinized and re-suspended in the medium. Male BALB/c nude mice (6-weeks-old) were subcutaneously inoculated in the dorsal area with $2.0 \times 10^{6}$ cells in $0.1 \mathrm{ml}$. Six weeks after inoculation, the mice were sacrificed and the tumors were dissected and fixed for CD34 immunohistochemical staining.

Statistical analysis. Data are expressed as the mean \pm SEM. Differences between groups were assessed using the t-test or Mann-Whitney U test. Time-to-recurrence was calculated according to the Kaplan-Meier method and compared using the log-rank test. All statistical analyses were performed using Prism GraphPad software (GraphPad Software, La Jolla, CA, USA). A P-value $<0.05$ denoted the presence of a statistically significant difference.

\section{Results}

Expression of miR-210 is upregulated in HBV-related HCC. In order to examine the expression of hypoxic miR-210 in HCC, miR-210 expression was measured by qRT-PCR in 212 paired HCC tumor and non-tumor samples obtained from surgery (Fig. 1A). miR-210 expression in HCC tissues was significantly higher than that in non-tumor tissues. In addition, to determine whether hepatitis stress induced miR-210 expression, 31 healthy liver samples obtained from HBV-negative hepatic hemangioma patients were examined, and we found that miR-210 expression level in healthy liver was significantly lower than that in the HCC non-tumor tissues (Fig. 1A). Thus, miR-210 expression was increased progressively from normal liver and adjacent non-tumor tissues, to HCC tissues.

We also determined whether miR-210 expression was correlated with the progression of liver disease. According to the pathological reports, we divided non-tumor samples into the hepatitis group (100 patients) and cirrhosis group (112 patients), the HCC samples were divided into the BCLC I+II group (33 patients) and the BCLC III+IV group 
Table I. Comparison of clinicopathological and demographic characteristics of patients with high and low miR-210 expression level.

\begin{tabular}{|c|c|c|c|}
\hline & $\begin{array}{l}\text { miR-210 high expression } \\
\qquad(\mathrm{n}=106)\end{array}$ & $\begin{array}{l}\text { miR-210 low expression } \\
\qquad(\mathrm{n}=106)\end{array}$ & P-value \\
\hline Gender & & & 0.369 \\
\hline Male & $97(91.51)$ & $93(87.73)$ & \\
\hline Female & $9(8.49)$ & $13(12.27)$ & \\
\hline Age (years) ${ }^{\mathrm{a}}$ & $49.77 \pm 10.63$ & $50.89 \pm 10.14$ & 0.936 \\
\hline Cirrhosis & & & 0.411 \\
\hline Yes & $51(48.11)$ & $57(53.77)$ & \\
\hline No & $55(51.89)$ & $49(46.23)$ & \\
\hline $\mathrm{HBeAg}$ & & & 0.434 \\
\hline Positive & $30(28.30)$ & $25(23.58)$ & \\
\hline Negative & $76(71.70)$ & $81(76.42)$ & \\
\hline $\operatorname{AFP}(\mathrm{ng} / \mathrm{ml})$ & & & 0.674 \\
\hline$\geq 20$ & $66(62.26)$ & $63(59.43)$ & \\
\hline$<20$ & $40(37.74)$ & $43(40.57)$ & \\
\hline Alanine aminotransferase (U/l) & & & 0.088 \\
\hline$\geq 40$ & $45(42.45)$ & $33(31.13)$ & \\
\hline$<40$ & $61(57.55)$ & $73(68.87)$ & \\
\hline Aspartate aminotransferase (U/l) & & & 0.059 \\
\hline$\geq 40$ & $42(39.62)$ & $29(27.36)$ & \\
\hline$<40$ & $64(60.38)$ & $77(72.64)$ & \\
\hline Total bilirubin (ummol/ml) & & & 0.465 \\
\hline$\geq 17.1$ & $37(34.90)$ & $32(30.19)$ & \\
\hline$<17.1$ & $69(65.10)$ & $74(69.81)$ & \\
\hline Albumin (g/l) & & & 0.361 \\
\hline$\geq 35$ & $73(68.87)$ & $79(74.52)$ & \\
\hline$<35$ & $33(31.13)$ & $27(25.48)$ & \\
\hline HBV DNA (IU/ml) & & & 0.321 \\
\hline$\geq 2,000$ & $63(59.43)$ & $70(66.04)$ & \\
\hline$<2,000$ & $43(40.57)$ & $36(33.96)$ & \\
\hline Tumor diameter $(\mathrm{cm})^{\mathrm{a}}$ & $7.28 \pm 4.07$ & $6.29 \pm 3.89$ & $0.035^{\mathrm{b}}$ \\
\hline Tumor encapsulation & & & $0.022^{\mathrm{b}}$ \\
\hline None & $76(71.70)$ & $60(56.60)$ & \\
\hline Complete & $30(28.30)$ & $46(43.40)$ & \\
\hline Vascular invasion & & & $0.008^{b}$ \\
\hline Yes & $52(49.06)$ & $33(31.13)$ & \\
\hline No & $54(50.94)$ & $73(68.87)$ & \\
\hline Tumor number & & & 0,208 \\
\hline Single & $83(78.30)$ & $75(70.75)$ & \\
\hline Multiple & $23(21.70)$ & $31(29.25)$ & \\
\hline Tumor differentiation (BCLC) & & & $0.014^{\mathrm{b}}$ \\
\hline $\mathrm{I} / \mathrm{II}$ & $10(9.43)$ & $23(21.70)$ & \\
\hline III/IV & $96(90.57)$ & $83(78.30)$ & \\
\hline
\end{tabular}

${ }^{\mathrm{a} A g e}$ and tumor diameter are expressed as mean $\pm \mathrm{SD}$. HBsAg, hepatitis B surface antigen; HBeAg, hepatitis B e-antigen; AFP, $\alpha$-fetoprotein; BCLC, Barcelona-Clinic Liver Cancer classification; ${ }^{\mathrm{P}}<0.05$. 
Table II. Univariate and multivariate analysis of factors associated with tumor-free survival of HCC patients.

\begin{tabular}{|c|c|c|c|c|}
\hline \multirow[b]{2}{*}{ Factors } & \multicolumn{2}{|c|}{ Univariate analysis } & \multicolumn{2}{|c|}{ Multivariate analysis } \\
\hline & $\mathrm{HR}(95 \% \mathrm{CI})$ & P-value & HR $(95 \% \mathrm{CI})$ & P-value \\
\hline Age (years) & $0.987(0.620-1.571)$ & 0.957 & - & - \\
\hline Gender & $0.688(0.359-1.316)$ & 0.258 & - & - \\
\hline Liver cirrhosis (yes vs. no) & $0.946(0.657-1.362)$ & 0.765 & - & - \\
\hline Tumor differentiation (III+IV vs. I+II) & $1.217(0.717-2.065)$ & 0.468 & - & - \\
\hline Tumor diameter ( $\geq 5$ vs. $<5 \mathrm{~cm})$ & $1.375(0.947-1.997)$ & 0.094 & - & - \\
\hline Tumor encapsulation (no vs. yes) & $1.374(0.938-2.015)$ & 0.103 & - & - \\
\hline Alanine aminotransferase ( $\geq 40$ vs. $<40 \mathrm{U} / \mathrm{l}$ ) & $1.393(0.964-2.012)$ & 0.078 & - & - \\
\hline Total bilirubin ( $\geq 17.1$ vs. $<17.1 \mathrm{ummol} / \mathrm{ml}$ ) & $1.014(0.852-1.206)$ & 0.879 & - & - \\
\hline $\operatorname{HBV}$ DNA $(\geq 2,000$ vs. $<2,000 \mathrm{IU} / \mathrm{ml})$ & $1.354(0.916-2.002)$ & 0.129 & - & - \\
\hline HBeAg (positive vs. negative) & $1.130(0.755-1.693)$ & 0.552 & - & - \\
\hline $\operatorname{AFP}(\geq 20$ vs. $<20 \mathrm{ng} / \mathrm{ml})$ & $1.323(0.895-1.956)$ & 0.161 & - & - \\
\hline Aspartate aminotransferase ( $\geq 40$ vs. $<40 \mathrm{U} / \mathrm{l})$ & $1.631(1.130-2.355)$ & $0.009^{\mathrm{a}}$ & $1.363(0.933-1.991)$ & 0.110 \\
\hline Tumor number (multiple vs. single) & $1.834(1.236-2.722)$ & $0.003^{\mathrm{a}}$ & $1.504(0.988-2.290)$ & 0.057 \\
\hline miR-210 expression (high vs. low) & $1.549(1.071-2.239)$ & $0.02^{\mathrm{a}}$ & $1.515(1.041-2.203)$ & $0.03^{\mathrm{a}}$ \\
\hline Vascular invasion (yes vs. no) & $1.963(1.345-2.865)$ & $<0.001^{\mathrm{a}}$ & $1.675(1.116-2.513)$ & $0.013^{\mathrm{a}}$ \\
\hline
\end{tabular}

HRs $(95 \% \mathrm{CI})$ and P-values were calculated using univariate and multivariate Cox proportional hazard regression. HBeAg, hepatitis B e-antigen; AFP, $\alpha$-fetoprotein; CI, confidence interval; ${ }^{\mathrm{P}}<0.05$.

The decrease of miR-210 in the cirrhosis group suggests that miR-210 expression may be correlated with liver damage (42), and the increase of miR-210 in the BCLC III+IV group suggests that miR-210 induction is correlated with HCC progression.

miR-210 expression is correlated with the prognosis of $H C C$ patients. To analyze the correlation between miR-210 expression and HCC prognosis, we divided the patients into two groups according to miR-210 expression in HCC using the median value as the cut-off. The clinicopathological and demographic characteristics of the patients in these two groups are listed in Table I. Compared to the miR-210 low group, miR-210 high group had significantly less tumor encapsulation $(\mathrm{P}=0.020)$, larger tumor size $(7.82 \pm 4.07$ vs. $6.29 \pm 3.89 \mathrm{~cm}$; $\mathrm{P}=0.035)$, more vascular invasion $(\mathrm{P}=0.008)$ and a higher proportion of undifferentiated tumors $(\mathrm{P}=0.014)$. Furthermore, patients with high miR-210 expression in HCC had significantly shorter tumor-free survival $(\mathrm{P}=0.036$; Fig. 1C) and overall survival $(\mathrm{P}=0.012$; Fig. $1 \mathrm{C})$, as compared to that of the miR-210 low patients. Moreover, multivariate analysis showed that HCC miR-210 expression ( $\mathrm{P}=0.03$; Table II) and vascular invasion $(\mathrm{P}=0.013$; Table II) were significant independent factors for tumor-free survival, and HCC miR-210 expression $(\mathrm{P}=0.012$; Table III) and tumor diameter $(\mathrm{P}=0.001$; Table III) were significant independent factors for overall survival. Thus, miR-210 expression is significantly correlated with the prognosis of HCC patients.

To further assess the possible prognostic value of miR-210, we evaluated miR-210 expression in non-tumor liver tissues. The expression of miR-210 in non-tumor tissues was not correlated with tumor-free survival $(\mathrm{P}=0.479)$ or overall survival ( $\mathrm{P}=0.290)$ of $\mathrm{HCC}$ patients (data not shown). However, the distribution of relative tumor/non-tumor (T/nT) miR-210 expression suggested the complicated regulation of miR-210 expression in the tumor microenvironment (Fig. 1D). According to the T/nT miR-210 expression fold-change, we used one fold as the cut-off value (35) and further divided all patients into 3 groups, T/nT $>2.0$ (91 patients), $\mathrm{T} / \mathrm{nT}<0.5$ (35 patients), and T/nT 0.5-2.0 (86 patients). The Kaplan-Meier analysis of both tumor-free and overall survival showed that the 3 groups of patients were clearly distinguished $(\mathrm{P}=0.0001$; Fig. 1E), suggesting that miR-210 expression $\mathrm{T} / \mathrm{nT}<0.5$ may serve as a favorable prognostic factor for HBV-related HCC. Thus, miR-210 expression in HCC, particularly the T/nT value, is a new prognosis predictor for HCC patients.

miR-210 promotes HCC angiogenesis. As the role of miR-210 in HCC angiogenesis remains unclear, we then investigated the correlation between miR-210 expression in HCC and intratumor MVD. The results of CD34 immunohistochemistry showed that HCC tissues with high miR-210 expression had high CD34 staining (Fig. 2A). In addition, miR-210 expression in HCC was positively correlated with the MVD CD34 value $(\mathrm{R} 2=0.1925 ; \mathrm{P}=0.0018 ;$ Fig. $2 \mathrm{~B})$, in a randomly selected group of $48 \mathrm{HCC}$ patients. To determine whether miR-210 is an independent angiogenesis inducer, we established miR-210 stably overexpressed HCC cell lines SMMC-7721 and HL-7702 and the corresponding control cell lines for in vivo and in vitro assays. The expression level of miR-210 was confirmed by qRT-PCR in each cell line (Fig. 2C and E), and we inoculated both control and miR-210 stably overexpressed SMMC-7721 cells into nude mice. CD34 staining 
Table III. Univariate and multivariate analysis of factors associated with overall survival of HCC patients.

\begin{tabular}{|c|c|c|c|c|}
\hline \multirow[b]{2}{*}{ Factors } & \multicolumn{2}{|c|}{ Univariate analysis } & \multicolumn{2}{|c|}{ Multivariate analysis } \\
\hline & $\mathrm{HR}(95 \% \mathrm{CI})$ & P-value & $\mathrm{HR}(95 \% \mathrm{CI})$ & P-value \\
\hline Age (years) & $1.029(0.597-1.774)$ & 0.918 & - & - \\
\hline Gender & $0.691(0.319-1.498)$ & 0.349 & - & - \\
\hline Liver cirrhosis (yes vs. no) & $0.927(0.606-1.419)$ & 0.728 & - & - \\
\hline Tumor differentiation (III+IV vs. I+II) & $1.987(0.994-3.974)$ & 0.052 & - & - \\
\hline Tumor encapsulation (no vs. yes) & $1.386(0.886-2.168)$ & 0.153 & - & - \\
\hline Alanine aminotransferase ( $\geq 40 \mathrm{vs}$. $<40 \mathrm{U} / \mathrm{l}$ ) & $1.345(0.876-2.066)$ & 0.176 & - & - \\
\hline Total bilirubin ( $\geq 17.1$ vs. $<17.1 \mathrm{ummol} / \mathrm{ml}$ ) & $0.863(0.619-1.205)$ & 0.387 & - & - \\
\hline HBV DNA ( $\geq 2,000$ vs. $<2,000 \mathrm{IU} / \mathrm{ml})$ & $1.447(0.911-2.300)$ & 0.118 & - & - \\
\hline HBeAg (positive vs. negative) & $1.069(0.661-1.727)$ & 0.786 & - & - \\
\hline Vascular invasion (yes vs. no) & $2.505(1.616-3.881)$ & $<0.001^{\mathrm{a}}$ & $1.365(0.916-2.213)$ & 0.163 \\
\hline $\operatorname{AFP}(\geq 20$ vs. $<20 \mathrm{ng} / \mathrm{ml})$ & $1.645(1.025-2.641)$ & $0.039^{\mathrm{a}}$ & $1.364(0.867-2.145)$ & 0.179 \\
\hline Aspartate aminotransferase ( $\geq 40 \mathrm{vs.}<40 \mathrm{U} / \mathrm{l}$ ) & $1.800(1.176-2.758)$ & $0.007^{\mathrm{a}}$ & $1.426(0.958-2.195)$ & 0.152 \\
\hline Tumor number (multiple vs. single) & $1.732(1.088-2.757)$ & $0.021^{\mathrm{a}}$ & $1.278(0.767-2.131)$ & 0.346 \\
\hline miR-210 expression (high vs. low) & $1.817(1.173-2.814)$ & $0.007^{\mathrm{a}}$ & $1.781(1.136-2.794)$ & $0.012^{\mathrm{a}}$ \\
\hline Tumor diameter $(\geq 5$ vs. $<5 \mathrm{~cm})$ & $1.575(1.015-2.446)$ & $0.043^{\mathrm{a}}$ & $2.181(1.349-3.527)$ & $0.001^{\mathrm{a}}$ \\
\hline
\end{tabular}

HRs $(95 \% \mathrm{CI})$ and P-values were calculated using univariate and multivariate Cox proportional hazard regression. HBeAg, hepatitis B e-antigen; AFP, $\alpha$-fetoprotein; CI, confidence interval; ${ }^{\text {P }}<0.05$.

of the xenograft showed that miR-210 stably overexpressed SMMC-7721 cells had higher CD34 staining as compared to that of control cells (Fig. 2D), suggesting that HCC cells with increased miR-210 may send a signal to recruit more endothelial cells for vascular formation. Moreover, we used the medium supernatants from control and miR-210 stably overexpressed HL-7702 cells for the tube formation assay, and the result showed that the supernatants from HL-7702 of increased miR-210 expression induced greater tube formation (Fig. 2F). Thus, we conclude that increased miR-210 expression in $\mathrm{HCC}$ promotes angiogenesis, which may be dependent on the secreted cytokines.

miR-210 targets FGFRL1 expression and secretion in HCC. We next investigated the target genes of miR-210, which are responsible for miR-210 mediated promotion of HCC angiogenesis. As the target genes of miR-210 have been studied in several genomic approaches $(8,43)$, we only screened for the experimentally proved target genes, which may regulate angiogenesis with extracellular secretion capability and which were relatively conserved between human and mouse. One of the target genes of miR-210, FGFRL1, was found to fulfill all the criteria listed above. FGFRL1, also known as FGFR5, and belongs to the fibroblast growth factor receptor (FGFR) family. FGFRs consist of an extracellular region of 3 immunoglobulin-like domains, a single hydrophobic membrane-spanning segment, and a cytoplasmic tyrosine kinase domain for cascading downstream signals. The extracellular portion of FGFRs binds to fibroblast growth factors (FGFs). However, as a decoy FGF receptor (44), FGFRL1 lacks the cytoplasmic tyrosine kinase domain (Fig. 3A).
It has been reported that FGFRL1 can be shed from the cell membrane and secreted in myoblasts and HEK293 cells (40), but it is unclear whether hepatocytes can secrete FGFRL1. We established an FGFRL1 stably transduced SMMC-7721 cell line to evaluate the shedding of FGFRL1 from hepatocytes. As previously mentioned, FGFRL1 binds to heparin (40), therefore, the cell culture supernatant was subjected to heparin purification and then was analyzed by WB, while the whole cell lysate was served as a control (Fig. 3B). We obtained a WB signal for shed FGFRL1 in the cell culture supernatant, with a lower molecular weight due to loss of transmembrane and intracellular domains. This finding showed that FGFRL1 is released from the cell membrane in a soluble form, suggesting that FGFRL1 acts as a secreted regulator of FGF signaling.

Next, we determined whether hypoxia treatment changed the expression of FGFRL1. SMMC-7721 cells were treated with DFX for different time periods. HIF1 $\alpha$ expression was induced while the expression of FGFRL1 declined (Fig. 3C). To assess whether the decrease of FGFRL1 following hypoxia was miR-210-dependent, we transiently transfected SMMC-7721 cells with miR-210 mimic, miR-210 inhibitor and their respective controls. The results showed that compared with mimic-control transfected cells, miR-210 mimic transfected cells had lower expression of FGFRL1, which is similar to the decrease seen in DFX-treated cells (Fig. 3D). miR-210 inhibitor transfected cells showed no decrease of FGFRL1 when treated with DFX (Fig. 3D). These results indicated that hypoxia-induced downregulation of FGFRL1 was mainly through miR-210. We further assessed whether expression of miR-210 downregulated FGFRL1 secretion. The heparin extract of the supernatant and whole cell lysate of miR-210 


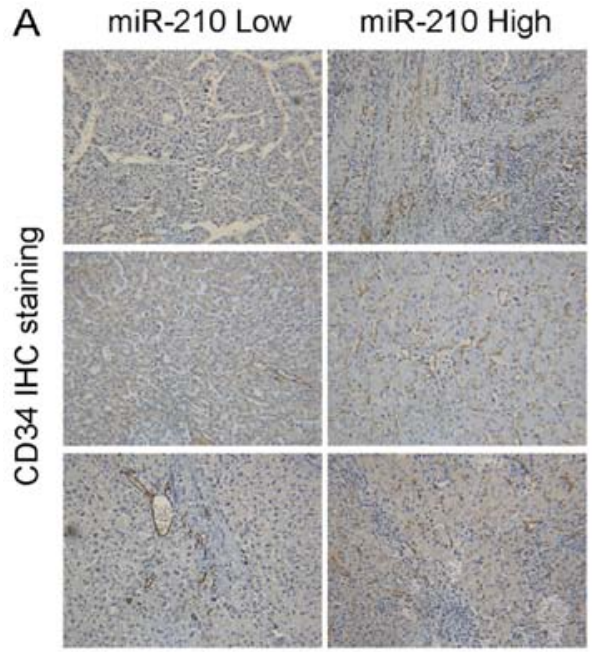

C

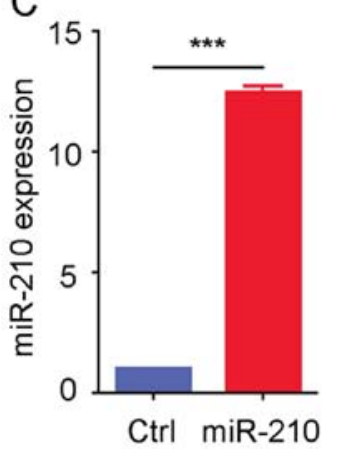

F

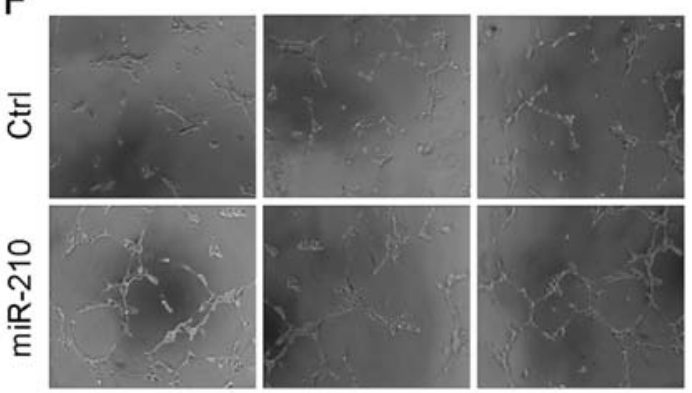

B

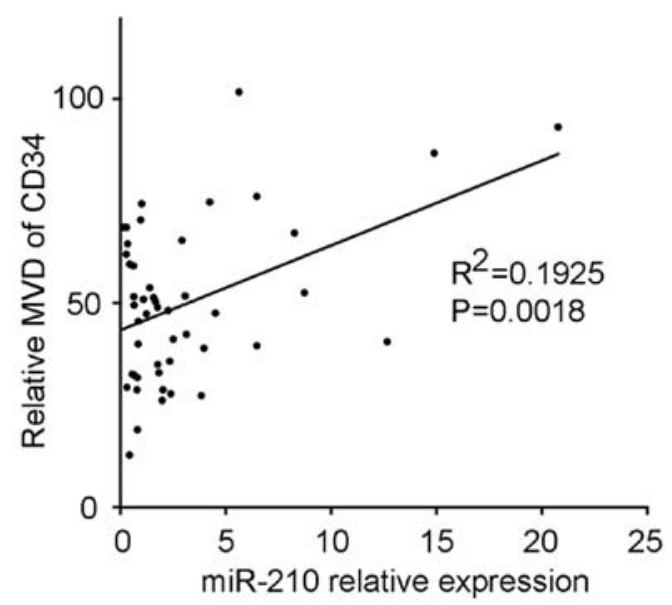

E

Figure 2. miR-210 promotes HCC angiogenesis both in vitro and in vivo. (A) Typical IHC CD34 staining results in HBV-related HCC tumor tissues show lower CD34 levels in samples with low miR-210 expression than that in samples with high miR-210 expression. (B) MVD CD34 levels in HCC tumor tissues are positively correlated with miR-210 expression level ( $2=0.1925 ; \mathrm{P}=0.0018)$. (C) miR-210 stably transfected SMMC-7721 cells showed higher miR-210 expression than that of control cells $(\mathrm{P}<0.001)$. (D) IHC CD34 staining assays show that ectopic miR-210 expression increases CD34 levels in nude mouse xenograft tumors. (E) miR-210 stably transfected HL-7702 cells showed higher miR-210 expression than that in control cells (P<0.001). (F) The supernatant from miR-210 stably overexpressed HL-7702 cells increases tube formation in HUVEC cells $\left(\mathrm{Nb}\right.$ nodes, ${ }^{*} \mathrm{P}<0.05 ;$ total segment length, ${ }^{* *} \mathrm{P}<0.01$. ImageJ angiogenesis-analyzer tool plugin).

stably transfected and control cells were examined by WB. The results showed that miR-210 expression downregulated FGFRL1 both inside and outside the cells (Fig. 3E). Hence, we conclude that hypoxia-induced miR-210 targets FGFRL1 expression and secretion in HCC.

FGFRL1 is a negative regulator of HCC angiogenesis. Although there are no reports on the angiogenesis functions of FGFRL1, we propose that FGFRL1 may inhibit angiogenesis by blocking FGF2 (45). To prove our hypothesis, we used a commercially available recombinant FGFRL1 protein in an in vitro angiogenesis assay. An in vitro HUVEC tube formation assay was performed to evaluate the effect of FGFRL1 on the anti-angiogenic response. HUVEC cells were seeded on Matrigel support using different stimulations: complete EGM2 medium without FGF2 (EGM2 - FGF2), complete EGM2 medium (EGM2), complete EGM2 medium with FGFRL1 (EGM2 + FGFRL1), and complete EGM2 medium with FGFRL1 plus FGF2 (EGM2 + FGFRL1 + FGF2). As shown in Fig. 4A, EGM2 + FGFRL1 significantly inhibited in vitro tube formation as compared to EGM2, while additional FGF2 rescued inhibition shown in the EGM2 + FGFRL1 + FGF2 group. Thus, FGFRL1 is a negative regulator of $\mathrm{HCC}$ angiogenesis, and hypoxia-induced miR-210 promotes HCC angiogenesis by targeting FGFRL1 expression and secretion.

Based on our findings, we propose the following working model to explain how induced miR-210 expression promotes 
A

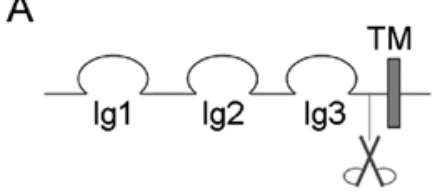

C

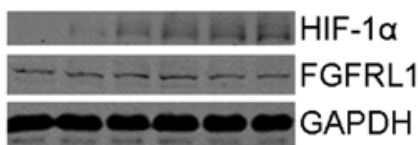

DFX $0 \begin{array}{llllll}0 & 1 & 2 & 3 & 4 & 6 \mathrm{~h}\end{array}$

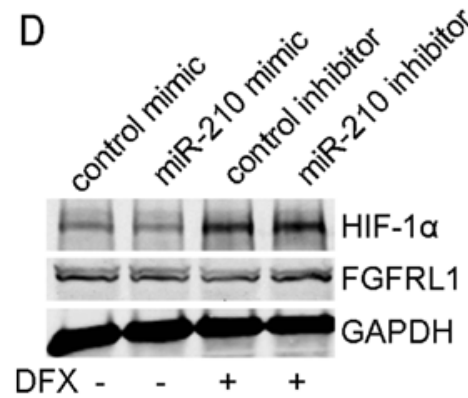

E

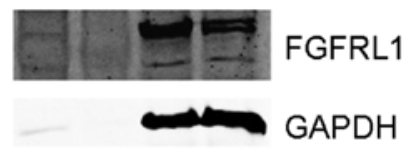

Supernatant Whole

heparin cell

purified lysate

Figure 3. miR-210 targets FGFRL1 expression and secretion in HCC. (A) The structure of FGFRL1. (B)ImageJ angiogenesis-analyzer western blot assays for FGFRL1 both inside and outside the cells. (C) Hypoxia stimulation due to DFX treatment decreases the expression of FGFRL1 in SMMC-7721 cells. (D) Western blot assays show that the transiently transfected miR210 mimic decreases the expression of FGFRL1, while the transfection of miR-210 inhibitor reversed the decrease of FGFRL1 by DFX. (E) miR-210 stably transfected SMMC-7721 cells have less FGFRL1 expression both outside and inside the cell.

HCC angiogenesis. Hypoxia induced the transcription factor HIF $1 \alpha$ expression and activation in HCC cells. HIF1 $\alpha$ then promotes the secretion of FGFs and VEGF to enhance HCC angiogenesis. Alternatively, HIF1 $\alpha$ induced the expression of hypoxic miR-210 to target FGFRL1 expression and secretion. As FGFRL1 is a decoy FGF receptor and an inhibitor of $\mathrm{HCC}$ angiogenesis, hypoxia-induced miR-210 promotes HCC angiogenesis by targeting FGFRL1 expression (Fig. 4B).

\section{Discussion}

In the present study, we demonstrated that miR-210 expression level was significantly upregulated in HCC tumor tissues, as compared to that of non-tumor liver tissues. In addition, miR-210 expression level altered with different stages of liver disease, and was correlated with prognosis of HCC patients. We also introduced a new working model of miR-210 promoted HCC angiogenesis through the targeting of FGFRL1. These data suggested that miR-210 may be an important regulator in HCC carcinogenesis and progression.

The relative expression level of $\mathrm{miR}-210 \mathrm{~T} / \mathrm{nT}$ value was determined to be a predictor of HCC prognosis. Previous studies suggested that miR-210 was not a specific HCC diagnostic biomarker (46), however, our data showed that miR-210 could be a predictor of HCC recurrence and final outcome, although more evidence from multicenter clinical studies are required to confirm these findings. Tumors with high expression of miR-210 showed more aggressive phenotypes, and the

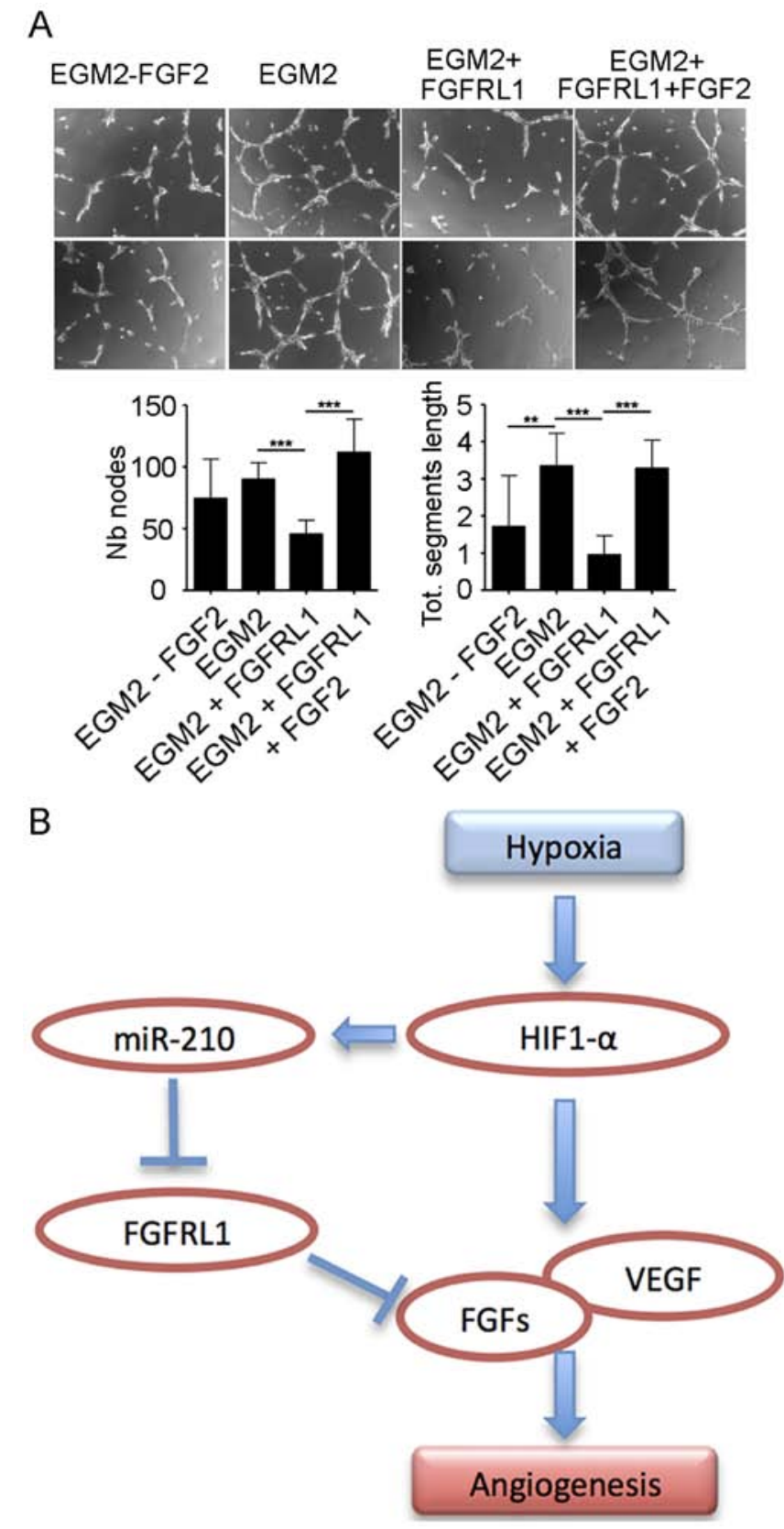

Figure 4. FGFRL1 is a negative regulator of HCC angiogenesis. (A) FGFRL1 decreases tube formation of HUVEC cells, and FGF2 reversed the decrease by FGFRL1 ( $\mathrm{Nb}$ nodes, $\mathrm{P}<0.01$; total segment length, $\mathrm{P}<0.01$. ImageJ angiogenesis-analyzer tool plugin). (B) Diagram of the working model.

expression of miR-210 was not always higher in tumor tissues. As miR-210 T/nT value classifies the prognosis of HCC patients more precisely, the regulation of miR-210 expression in HCC cells may be more complicated under hypoxic conditions. For example, the epigenetic regulation of miR-210 expression may be interesting, considering that the high GC content in the promoter region of miR-210 (43).

The induction of angiogenesis is a hallmark of cancer (3). Hypoxia regulates angiogenesis through different mechanisms (47), and miR-210 plays an important role in these mechanisms. It was reported that increased miR-210 in endothelial cells induced angiogenesis through activation of Notch signaling (19), and miR-210 could be exported out of 
the cell through exosome mediated mechanism to enhance angiogenesis $(22,23)$. In the present study, we describe a new mechanism for enhanced angiogenesis by miR-210 through downregulation of FGFRL1. As miRNAs can target multiple mRNAs, it is not surprising to find several angiogenesis associated mechanisms which miR-210 may use (15-24). Possible synchronization of different pro-angiogenesis mechanisms of miR-210 could be an economic way for cells to deal with hypoxic stress. Considering the other oncogenic effects of miR-210, those tumor cells with better vascular formation and a high expression level of miR-210 adapt better.

It is proposed that miR-210 can act both as an oncogene and a tumor suppressor, depending on specific cellular conditions (30). In general, our data suggest that miR-210 acts as an oncogene in the latter stages, at least in HCC. The genetic events which occurred in HCC cells during hypoxia adaptation are essential in the functional switch of miR-210. Those HCC cells which overcome hypoxic stress and maintain expression of miR-210 are more aggressive. In summary, the present study show higher miR-210 expression in HCC, which is correlated with poor prognosis of HCC patients, and hypoxic miR-210 promotes HCC angiogenesis.

\section{Acknowledgements}

We thank Dr Qiang Deng and Dr Yuan Gao from the Institute Pasteur of Shanghai for extensive assistance in our research projects, and Zhide Zhang for the excellent technical assistance. The present study was supported by the founding of National Key Basic Research Program of China (2014CB542102), the National High Technology Research and Development Program of China (2013AA032202), the Science Fund for Creative Research Groups, NSFC, China (81221061 and 81372207), the National Natural Science Foundation of China (81502416 and 81572791), the Innovation Program of Shanghai Municipal Education Commission (11ZZ76), and the Shanghai Municipal Commission of Health and Family Planning Project (140822111537488).

\section{References}

1. El-Serag HB: Hepatocellular carcinoma. N Engl J Med 365: 1118-1127, 2011.

2. Ott JJ, Stevens GA, Groeger J and Wiersma ST: Global epidemiology of hepatitis B virus infection: New estimates of age-specific HBsAg seroprevalence and endemicity. Vaccine 30: 2212-2219, 2012.

3. Hanahan D and Weinberg RA: Hallmarks of cancer: The next generation. Cell 144: 646-674, 2011.

4. Carmeliet P and Jain RK: Angiogenesis in cancer and other diseases. Nature 407: 249-257, 2000.

5. Chan YC, Banerjee J, Choi SY and Sen CK: miR-210: The master hypoxamir. Microcirculation 19: 215-223, 2012.

6. Giannakakis A, Sandaltzopoulos R, Greshock J, Liang S, Huang J, Hasegawa K, Li C, O'Brien-Jenkins A, Katsaros D, Weber BL, et al: miR-210 links hypoxia with cell cycle regulation and is deleted in human epithelial ovarian cancer. Cancer Biol Ther 7: 255-264, 2008.

7. Zhang Z, Sun H, Dai H, Walsh RM, Imakura M, Schelter J, Burchard J, Dai X, Chang AN, Diaz RL, et al: MicroRNA miR-210 modulates cellular response to hypoxia through the MYC antagonist MNT. Cell Cycle 8: 2756-2768, 2009.

8. Tsuchiya S, Fujiwara T, Sato F, Shimada Y, Tanaka E, Sakai Y, Shimizu K and Tsujimoto G: MicroRNA-210 regulates cancer cell proliferation through targeting fibroblast growth factor receptor-like 1 (FGFRL1). J Biol Chem 286: 420-428, 2011.
9. Biswas S, Roy S, Banerjee J, Hussain SR, Khanna S, Meenakshisundaram G, Kuppusamy P, Friedman A and Sen CK: Hypoxia inducible microRNA 210 attenuates keratinocyte proliferation and impairs closure in a murine model of ischemic wounds. Proc Natl Acad Sci USA 107: 6976-6981, 2010.

10. Kim HW, Haider HK, Jiang S and Ashraf M: Ischemic preconditioning augments survival of stem cells via miR-210 expression by targeting caspase-8-associated protein 2 . J Biol Chem 284: 33161-33168, 2009.

11. Hu S, Huang M, Li Z, Jia F, Ghosh Z, Lijkwan MA, Fasanaro P, Sun N, Wang X, Martelli F, et al: MicroRNA-210 as a novel therapy for treatment of ischemic heart disease. Circulation 122 (Suppl 11): S124-S131, 2010.

12. Crosby ME, Kulshreshtha R, Ivan M and Glazer PM: MicroRNA regulation of DNA repair gene expression in hypoxic stress. Cancer Res 69: 1221-1229, 2009.

13. Cicchillitti L, Di Stefano V, Isaia E, Crimaldi L, Fasanaro P, Ambrosino V, Antonini A, Capogrossi MC, Gaetano C, Piaggio G, et al: Hypoxia-inducible factor 1- $\alpha$ induces miR-210 in normoxic differentiating myoblasts. J Biol Chem 287: 44761-44771, 2012.

14. Gong Y, Xu F, Zhang L, Qian Y, Chen J, Huang H and Yu Y: MicroRNA expression signature for Satb2-induced osteogenic differentiation in bone marrow stromal cells. Mol Cell Biochem 387: 227-239, 2014.

15. Pulkkinen K, Malm T, Turunen M, Koistinaho $J$ and Ylä-Herttuala S: Hypoxia induces microRNA miR-210 in vitro and in vivo ephrin-A3 and neuronal pentraxin 1 are potentially regulated by miR-210. FEBS Lett 582: 2397-2401, 2008.

16. Alaiti MA, Ishikawa M, Masuda H, Simon DI, Jain MK, Asahara T and Costa MA: Up-regulation of miR-210 by vascular endothelial growth factor in ex vivo expanded CD $34^{+}$ cells enhances cell-mediated angiogenesis. J Cell Mol Med 16: 2413-2421, 2012.

17. Donnem T, Fenton CG, Lonvik K, Berg T, Eklo K, Andersen S, Stenvold H, Al-Shibli K, Al-Saad S, Bremnes RM, et al: MicroRNA signatures in tumor tissue related to angiogenesis in non-small cell lung cancer. PLoS One 7: e29671, 2012.

18. Liu F, Lou YL, Wu J, Ruan QF, Xie A, Guo F, Cui SP, Deng ZF and Wang Y: Upregulation of microRNA-210 regulates renal angiogenesis mediated by activation of VEGF signaling pathway under ischemia/perfusion injury in vivo and in vitro. Kidney Blood Press Res 35: 182-191, 2012.

19. Lou YL, Guo F, Liu F, Gao FL, Zhang PQ, Niu X, Guo SC, Yin JH, Wang Y and Deng ZF: miR-210 activates notch signaling pathway in angiogenesis induced by cerebral ischemia. Mol Cell Biochem 370: 45-51, 2012.

20. Shoji T, Nakasa T, Yamasaki K, Kodama A, Miyaki S, Niimoto T, Okuhara A, Kamei N, Adachi N and Ochi M: The effect of intra-articular injection of microRNA-210 on ligament healing in a rat model. Am J Sports Med 40: 2470-2478, 2012.

21. Yamasaki K, Nakasa T, Miyaki S, Yamasaki T, Yasunaga Y and Ochi M: Angiogenic microRNA-210 is present in cells surrounding osteonecrosis. J Orthop Res 30: 1263-1270, 2012.

22. Kosaka N, Iguchi H, Hagiwara K, Yoshioka Y, Takeshita F and Ochiya T: Neutral sphingomyelinase 2 (nSMase2)-dependent exosomal transfer of angiogenic microRNAs regulate cancer cell metastasis. J Biol Chem 288: 10849-10859, 2013.

23. Tadokoro H, Umezu T, Ohyashiki K, Hirano T and Ohyashiki JH: Exosomes derived from hypoxic leukemia cells enhance tube formation in endothelial cells. J Biol Chem 288: 34343-34351, 2013.

24. Zeng L, He X, Wang Y, Tang Y, Zheng C, Cai H, Liu J, Wang Y, Fu Y and Yang GY: MicroRNA-210 overexpression induces angiogenesis and neurogenesis in the normal adult mouse brain. Gene Ther 21: 37-43, 2014.

25. Chan SY, Zhang YY, Hemann C, Mahoney CE, Zweier JL and Loscalzo J: MicroRNA-210 controls mitochondrial metabolism during hypoxia by repressing the iron-sulfur cluster assembly proteins ISCU1/2. Cell Metab 10: 273-284, 2009.

26. Chen Z, Li Y,Zhang H, Huang P and Luthra R: Hypoxia-regulated microRNA-210 modulates mitochondrial function and decreases ISCU and COX10 expression. Oncogene 29: 4362-4368, 2010.

27. Puisségur MP, Mazure NM, Bertero T, Pradelli L, Grosso S, Robbe-Sermesant K, Maurin T, Lebrigand K, Cardinaud B, Hofman V, et al: miR-210 is overexpressed in late stages of lung cancer and mediates mitochondrial alterations associated with modulation of HIF-1 activity. Cell Death Differ 18: 465-478, 2011. 
28. Ying Q, Liang L, Guo W, Zha R, Tian Q, Huang S, Yao J, Ding J, Bao M, Ge C, et al: Hypoxia-inducible microRNA-210 augments the metastatic potential of tumor cells by targeting vacuole membrane protein 1 in hepatocellular carcinoma. Hepatology 54: 2064-2075, 2011.

29. Hong L, Han Y, Zhang H, Zhao Q and Qiao Y: miR-210: A therapeutic target in cancer. Expert Opin Ther Targets 17: 21-28, 2013.

30. Qin Q, Furong W and Baosheng L: Multiple functions of hypoxia-regulated miR-210 in cancer. J Exp Clin Cancer Res 33: $50,2014$.

31. Leung AK and Sharp PA: MicroRNA functions in stress responses. Mol Cell 40: 205-215, 2010.

32. Esquela-Kerscher A and Slack FJ: Oncomirs - microRNAs with a role in cancer. Nat Rev Cancer 6: 259-269, 2006.

33. Haybaeck J, Zeller N and Heikenwalder M: The parallel universe: microRNAs and their role in chronic hepatitis, liver tissue damage and hepatocarcinogenesis. Swiss Med Wkly 141: w13287, 2011.

34. Meng F, Henson R, Wehbe-Janek H, Ghoshal K, Jacob ST and Patel T: MicroRNA-21 regulates expression of the PTEN tumor suppressor gene in human hepatocellular cancer. Gastroenterology 133: 647-658, 2007.

35. Hou J, Lin L, Zhou W, Wang Z, Ding G, Dong Q, Qin L, Wu X, Zheng Y, Yang Y, et al: Identification of miRNomes in human liver and hepatocellular carcinoma reveals miR-199a/b-3p as therapeutic target for hepatocellular carcinoma. Cancer Cell 19: 232-243, 2011.

36. Pineau P, Volinia S, McJunkin K, Marchio A, Battiston C, Terris B, Mazzaferro V, Lowe SW, Croce CM and Dejean A miR-221 overexpression contributes to liver tumorigenesis. Proc Natl Acad Sci USA 107: 264-269, 2010

37. Zhan M, Li Y, Hu B, He X, Huang J, Zhao Y, Fu S and Lu L: Serum microRNA-210 as a predictive biomarker for treatment response and prognosis in patients with hepatocellular carcinoma undergoing transarterial chemoembolization. J Vasc Interv Radiol 25: 1279-1287.e1, 2014.
38. Yang W, Sun T, Cao J, Liu F, Tian Y and Zhu W: Downregulation of miR-210 expression inhibits proliferation, induces apoptosis and enhances radiosensitivity in hypoxic human hepatoma cells in vitro. Exp Cell Res 318: 944-954, 2012.

39. Yang W, Wei J, Sun T and Liu F: Effects of knockdown of miR-210 in combination with ionizing radiation on human hepatoma xenograft in nude mice. Radiat Oncol 8: 102, 2013

40. Steinberg F, Zhuang L, Beyeler M, Kälin RE, Mullis PE, Brändli AW and Trueb B: The FGFRL1 receptor is shed from cell membranes, binds fibroblast growth factors (FGFs), and antagonizes FGF signaling in Xenopus embryos. J Biol Chem 285: 2193-2202, 2010.

41. Weidner N, Semple JP, Welch WR and Folkman J: Tumor angiogenesis and metastasis - correlation in invasive breast carcinoma. N Engl J Med 324: 1-8, 1991.

42. Albanis E and Friedman SL: Hepatic fibrosis. Pathogenesis and principles of therapy. Clin Liver Dis 5: 315-334, v-vi, 2001.

43. Huang X, Ding L, Bennewith KL, Tong RT, Welford SM, Ang KK, Story M, Le QT and Giaccia AJ: Hypoxia-inducible mir-210 regulates normoxic gene expression involved in tumor initiation. Mol Cell 35: 856-867, 2009.

44. Bertrand S, Somorjai I, Garcia-Fernandez J, Lamonerie T and Escriva H: FGFRL1 is a neglected putative actor of the FGF signalling pathway present in all major metazoan phyla. BMC Evol Biol 9: 226, 2009.

45. Trueb B, Zhuang L, Taeschler $\mathrm{S}$ and Wiedemann $\mathrm{M}$ : Characterization of FGFRL1, a novel fibroblast growth factor (FGF) receptor preferentially expressed in skeletal tissues. J Biol Chem 278: 33857-33865, 2003.

46. Lu J, Xie F, Geng L, Shen W, Sui C and Yang J: Potential role of microRNA-210 as biomarker in human cancers detection: A meta-analysis. Biomed Res Int 2015: 303987, 2015.

47. Pugh CW and Ratcliffe PJ: Regulation of angiogenesis by hypoxia: Role of the HIF system. Nat Med 9: 677-684, 2003. 